\title{
Oxymatrine Inhibits the Proliferation and Invasion of Breast Cancer Cells via the PI3K Pathway
}

This article was published in the following Dove Press journal:

Cancer Management and Research

\author{
Lin Guo' \\ Tengfei Yang ${ }^{2}$ \\ 'Department of Gastrointestinal and \\ Nutriology Surgery, Shengjing Hospital of \\ China Medical University, Shenyang, \\ LiaoNing 11 0004, People's Republic of \\ China; ${ }^{2}$ The Department of Social \\ Service, Shengjing Hospital of China \\ Medical University, Shenyang, LiaoNing \\ I 10004, People's Republic of China
}

Purpose: Oxymatrine has been reported to possess anti-cancer activity, but its role in breast cancer (BC) is weakly defined. We investigated the anti-cancer effects of oxymatrine in human $\mathrm{BC}$ cells, and the underlying molecular mechanisms of these effects.

Methods: BC lines were treated with oxymatrine. The MTT assay was conducted to evaluate cell viability. The cell cycle and apoptosis of BC cells were analyzed using flow cytometry and Hoechst 33258 staining. Transwell ${ }^{\mathrm{TM}}$ assays were undertaken to measure the migratory and invasive abilities of MCF-7 or MDA-MB-231 cells. Expression of phosphatidylinositol 3-kinase (PI3K), Akt, cyclin D1, cluster of differentiation (CD)K2, PARP, Gsk3 $\beta$, caspase-3, matrix metalloproteinase (MMP)2 and Bax at protein and RNA levels was measured by Western blotting and quantitative real-time polymerase chain reaction.

Results: Oxymatrine inhibited the proliferation of BC cells in a time-dependent manner. It induced apoptosis in a dose- and time-dependent way according to Annexin V and Hoechst 33258 staining. Oxymatrine could inhibit the invasion of BC cells as shown by the Transwell assay. Oxymatrine inhibited expression of B-cell lymphoma-2 while increasing that of Bax as well as increasing expression of caspase- 3 and caspase-9. Addition of oxymatrine to BC cells attenuated the PI3K/Akt signaling pathway cascade, as evidenced by dephosphorylation of $\mathrm{P} 13 \mathrm{~K}$ and Akt.

Conclusion: Oxymatrine exerts its anti-tumor effects in BC cells by abolishing the PI3K pathway. Oxymatrine may be a new compound for BC treatment.

Keywords: oxymatrine, breast cancer, PI3K/Akt, proliferation, apoptosis, invasion

\section{Introduction}

Breast cancer (BC) is a major cause of cancer-related death for women. The mortality arising from $\mathrm{BC}$ is attributed to metastatic spread of cancer cells to vital organs, such as the liver, bone and lung. ${ }^{1}$ An estimated 2.1 million new cases of BC worldwide were recorded during $2018 .^{2}$

Breast tumors are characterized by their biologic complexity and heterogeneity. Progression of BC cells is a multi-step process that involves the dysregulation of the multiple genes that control cell survival. Oncology is focusing increasingly on finding important signaling pathways and targeting the molecules that promote the survival, proliferation and metastasis of tumor cells.

In addition to several types of surgical procedures, current treatment for BC requires judiciously applied serial endocrine, chemotherapeutic and biologic therapies. Surgery is the primary treatment for patients with early BC and improves long-term survival, but it is not efficacious for individuals with advanced BC. ${ }^{3}$ Non-surgical treatments for $\mathrm{BC}$ have been investigated. However, traditional non-surgical therapies 
are associated with significant toxicity. Therefore, the development of novel treatments is required urgently.

Natural products play an important part in cancer treatment. For example, a bitter-melon extract has been used for the treatment of $\mathrm{BC}$ or head and neck cancer. ${ }^{4-6}$ Oxymatrine (Figure 1A) is an alkaloid extracted from a traditional Chinese herb. Oxymatrine has been reported to inhibit the proliferation, cell cycle and angiogenesis of cancer cells, promote the apoptosis of cancer cells, and reverse multidrug resistance in patients with cancer. ${ }^{7}$ Some studies have reported the anti-cancer activity of oxymatrine in the pancreatic cancer cells, ${ }^{8}$ colon cancer cells,${ }^{9}$ hepatoma cells, ${ }^{10}$ gastric cancer cells ${ }^{11}$ and osteosarcoma cells of humans. ${ }^{12}$ However, reports of the anti-cancer activity of oxymatrine on human BC cells are lacking, a knowledge gap that we sought to fill in the present study.

\section{Materials and Methods}

\section{Reagents}

Dulbecco's modified Eagle's medium (DMEM)-high glucose was purchased from Gibco (Gaithersburg, MD, USA). 3-(4,5-Dimethylthiazol-2-yl)-2,5-diphenyltetrazolium bromide (MTT) was purchased from Sigma-Aldrich (Saint Louis, MO, USA). Rabbit polyclonal antibodies against glyceraldehyde 3-phosphate dehydrogenase (GAPDH) and caspase-3 and monoclonal antibodies against Bax and B-cell lymphoma (Bcl)-2 (Abcam, Cambridge, UK) were used at 1:5000 dilution. Rabbit monoclonal antibody to phosphatidylinositol 3-kinase (PI3K), Akt, and Aktp-Thr308 (Santa Cruz Biotechnology, Santa Cruz, CA, USA) was used at 1:1000 dilution. Rabbit monoclonal antibody to cyclin D1, cluster of differentiation (CD)K2, Gsk $3 \beta$ and matrix metalloproteinase (MMP)-2 (Proteintech, Chicago, IL, USA) was used at 1:1000 dilution.

\section{Cell Culture}

The BC lines MCF-7 and MDA-MB-231 and human embryonic kidney (HEK)-293 cells (used as controls) were purchased from the Cell Bank of the Chinese Academy of Sciences (Shanghai, China). Cells were cultured in DMEM containing $10 \%$ fetal bovine serum, $100 \mathrm{U} / \mathrm{mL}$ penicillin and $100 \mu \mathrm{g} / \mathrm{mL}$ streptomycin. All cell types were grown at $37^{\circ} \mathrm{C}$ in a humidified incubator in an atmosphere of $5 \% \mathrm{CO}_{2}$.

\section{MTT Assay}

MTT assays were conducted to evaluate cell viability, as described previously. Briefly, MCF-7, MDA-MB-231 or
HEK-293 cells were seeded at $10^{4} /$ well in 96 -well plates and plated in $0.1 \mathrm{~mL}$ DMEM supplemented with indicated concentrations of oxymatrine for 12, 24, 36 or $48 \mathrm{hrs}$. At each time point, $10 \mu \mathrm{L}$ of MTT solution $(5 \mathrm{mg} / \mathrm{mL})$ was added, followed by incubation for $4 \mathrm{hrs}$ at $37^{\circ} \mathrm{C}$. Then, the medium was replaced by $150 \mu \mathrm{L}$ of dimethyl sulfoxide (DMSO) solution, followed by incubation for another 10 mins to solubilize crystals. The absorbance was read at $490 \mathrm{~nm}$ using a microplate reader (Bio-Rad Laboratories, Hercules, CA, USA).

\section{Hoechst 33258 Staining}

MCF-7 cells were treated with the indicated concentrations of oxymatrine for $24 \mathrm{hrs}$. After incubation, cells were fixed with $4 \%$ polyoxymethylene and washed thrice with phosphatebuffered saline (PBS), followed by incubation with Hoechst $33258(10 \mu \mathrm{g} / \mathrm{mL})$ in the dark for another 5 mins and washed thrice with PBS. Cells were observed and photographed under a fluorescence microscope.

\section{Flow Cytometry}

MCF-7 cells were cultured with the indicated concentrations of oxymatrine for $24 \mathrm{hrs}$ and then apoptosis was measured using the Annexin V-FITC Apoptosis Detection kit. Cells were collected by trypsinization, centrifuged at $1000 \times \mathrm{g}$ for $5 \mathrm{mins}$ at room temperature, resuspended in $195 \mu \mathrm{L}$ of Annexin V-FITC Binding Buffer and mixed with $5 \mu \mathrm{L}$ of Annexin V-FITC. Then, cells were stained in the dark for 10 mins. After that, cells were centrifuged at $1000 \times \mathrm{g}$ for an additional $5 \mathrm{mins}$, resuspended in $190 \mu \mathrm{L}$ of Annexin V-FITC Binding Buffer and mixed with $10 \mu \mathrm{L}$ of propidium iodide. Then, cells were kept in the dark and subjected to flow cytometry. Experiments were repeated thrice, and the results were analyzed using CellQuest ${ }^{\mathrm{TM}}$ (Becton Dickinson, Franklin Lakes, NJ, USA).

\section{Assays to Measure the Migration and Invasion of Cells}

Transwell ${ }^{\mathrm{TM}}$ assays (Sigma-Aldrich) were undertaken to measure the migratory and invasive abilities of MCF-7 and MDA-MB-231 cells. The upper chambers were washed with serum-free medium, with or without the addition of $20 \mu \mathrm{L}$ of Matrigel ${ }^{\mathrm{TM}}$ (Corning Life Sciences, Corning, NY, USA) covering the surface of a polycarbonate membrane for migration or invasion experiments. Cells $\left(10^{5}\right)$ in $0.2 \mathrm{~mL}$ of serum-free DMEM treated with or without the 
A<smiles>O=C1CCCC2CCC[N+]([O-])([O-])C12</smiles>

oxymatrine
B

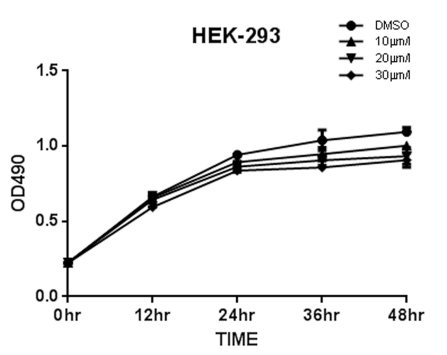

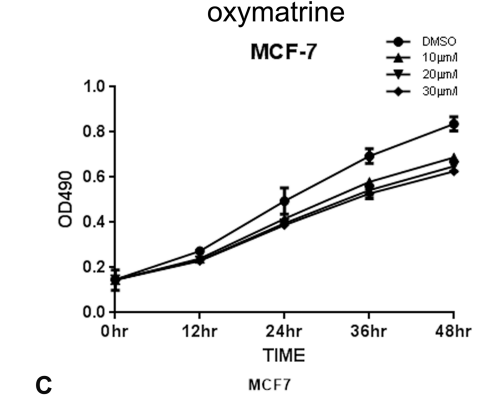
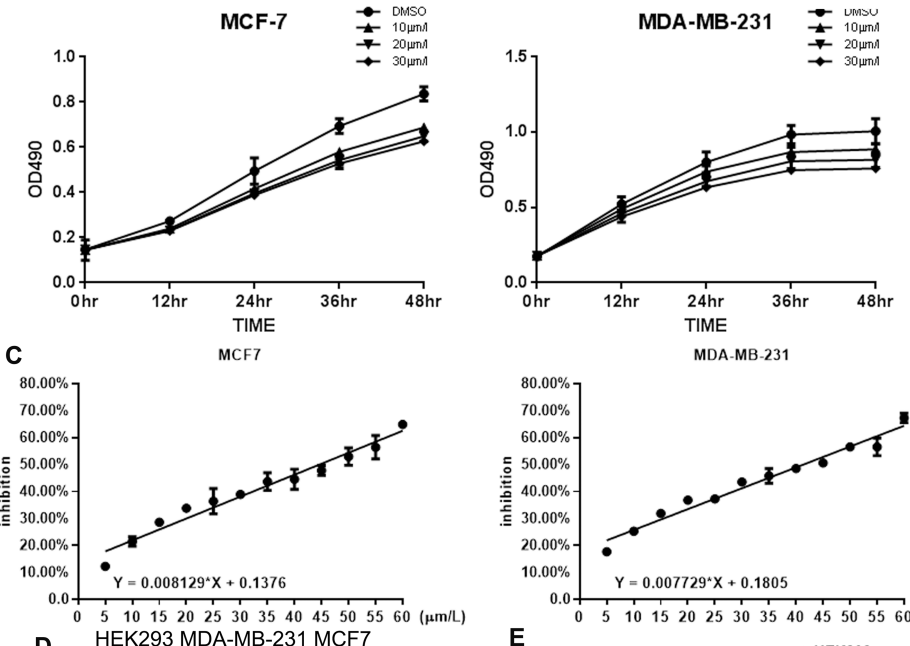

D HEK293 MDA-MB-231 MCF7
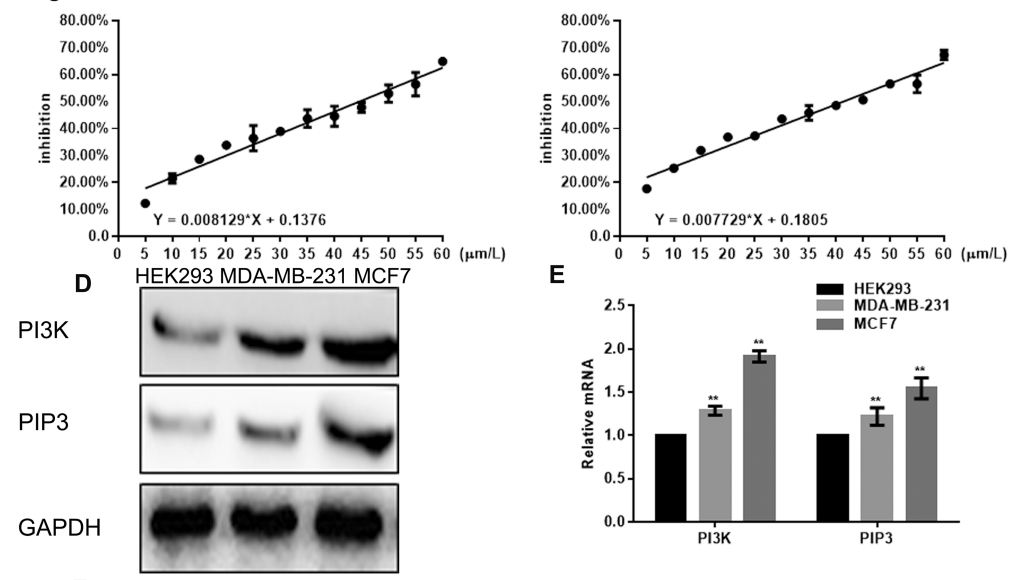

G

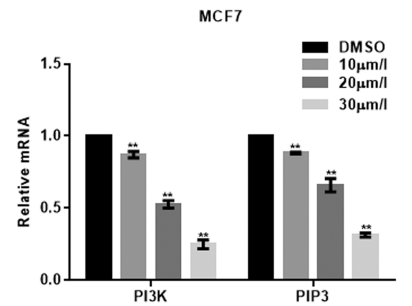

GAPDH
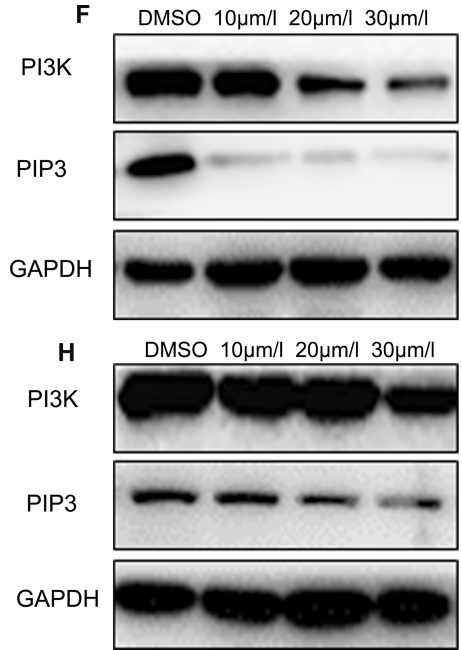

I

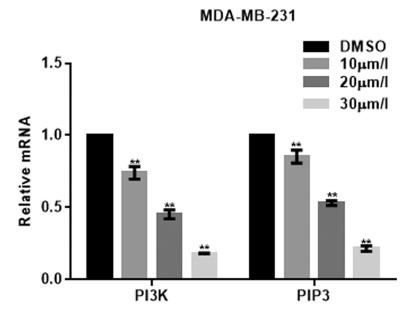

Figure I Oxymatrine inhibits the proliferation of breast cancer cells. (A) Molecular structure of oxymatrine. (B) HEK-293, MCF-7 and MDA-MB-23I cells were cultured with the indicated concentrations of oxymatrine for the indicated times in 96-well plates. The MTT assay was carried out, and results are the mean \pm SD of three experiments done in triplicate. (C) MCF-7 and MDA-MB-23I cells were cultured with the indicated concentrations of oxymatrine for the indicated times in 96-well plates. The MTT assay was carried out to calculate the inhibition of cell proliferation by oxymatrine, and the results are the mean \pm SD of three experiments done in triplicate. (D) HEK-293, MCF-7 and MDA-MB-23I cells were cultured with the indicated concentrations of oxymatrine for $24 \mathrm{hrs}$, and PI3K expression was measured by Western blotting. (E) HEK-293, MCF-7 and MDA-MB-23I cells were cultured with the indicated concentrations of oxymatrine for 24 hrs, and PI3K expression was measured by real-time RT-PCR. (F) MCF-7 cells were treated with DMSO alone or with the indicated concentrations of oxymatrine for $24 \mathrm{hrs}$, and PI3K expression was measured by Western blotting. (G) MCF-7 cells were treated with DMSO alone or the indicated concentrations of oxymatrine for $24 \mathrm{hrs}$, and PI3K expression was measured by real-time RT-PCR. Results represent the mean \pm SD of three experiments done in triplicate. (H) MDA-MB-23 I cells were treated with DMSO alone or the indicated concentrations of oxymatrine for 24 hrs, and PI3K expression was measured by Western blotting. (I) MDA-MB-23I cells were treated with DMSO alone or the indicated concentrations of oxymatrine for $24 \mathrm{hrs}$, and PI3K expression was measured by real-time RT-PCR. Results are the mean \pm SD of three experiments done in triplicate. $* * \mathrm{P}<0.01$, compared with the control group. 
indicated doses of oxymatrine were plated in the upper chamber of each chamber, whereas the lower surfaces were filled with $0.5 \mathrm{~mL}$ of DMEM supplemented with $10 \%$ fetal bovine serum. After incubation for $24 \mathrm{hrs}$ at $37^{\circ} \mathrm{C}$, cells on the upper compartments were removed, whereas the invaded cells in the lower parts were stained, observed and counted under a high-power microscope.

\section{Western Blotting}

Lysates of total cellular proteins were extracted using $100 \mu \mathrm{L}$ of RIPA Lysis Buffer. Then, $60 \mu \mathrm{g}$ of lysates resolved in gels after sodium dodecyl sulfate-polyacrylamide gel electrophoresis was transferred to nitrocellulose membranes through electroblotting. Then, membranes were blocked with 5\% blocking solution for $1 \mathrm{hr}$. This was followed by incubation overnight at $4^{\circ} \mathrm{C}$ with primary antibodies obtained from Santa Cruz Biotechnology (PI3K; 1:1000 dilution; sc-390916), Akt (1:1000; sc-5298), Akt ${ }^{\mathrm{p}-\mathrm{Tyr} 308}(1: 1000 ; \mathrm{sc}-271966)$, caspase9 (1:1000; sc-56076), Bax (1:1000; sc-7480), Bcl-2 (1:1000; sc-7382), GAPDH (1:1000; sc-47724), caspase-3 (1:1000; sc7272), PARP (1:1000; sc-390771), cyclin D1 (1:1000; sc-8396), CDK2 (1:1000; sc-6248), GSK-3 3 (1:1000; sc377213), MMP2 (1:1000; sc-13594) apart from PIP3 (1:800; PAB22210), which was from Abnova (Taipei, Taiwan). Then, membranes were washed thrice with TBST and incubated with horseradish peroxidase-conjugated secondary antibodies (Sigma-Aldrich) for an additional hour. Immunoreactivity was measured using Western Lighting Ultra (Pierce Technology, Rockford, IL, USA).

\section{Quantitative Real-Time Polymerase Chain Reaction (PCR)}

Total cellular RNA was extracted by $1 \mathrm{~mL}$ of TRIzol ${ }^{\mathrm{TM}}$ Reagent according to the manufacturer (Invitrogen, Carlsbad, CA, USA) protocols. Then, $1 \mathrm{mg}$ of RNA was reversetranscribed to cDNA in a $20-\mu \mathrm{L}$ system by an RT Reaction kit (Promega, Fitchburg, WI, USA). Real-time PCR was done using an Mx3000P Real-Time PCR system (Applied Biosystems, Foster City, CA, USA). The PCR program was: 40 cycles of $94^{\circ} \mathrm{C}$ for $15 \mathrm{~s}, 60^{\circ} \mathrm{C}$ for $10 \mathrm{~s}$ and $72^{\circ} \mathrm{C}$ for $20 \mathrm{~s}$. All procedures were repeated thrice. Gene expression was normalized to that of $\beta$-actin.

The mouse primer sequences (forward and reverse, respectively) used were: PI3K, 5'- GGACCCGATGCGGTTAGAG$3^{\prime}$ and 5'-ATCAAGTGGATGCCCCACAG-3'; Gsk3 $\beta$, 5'-GT ATGGTCTGCTGGCTGTGT-3' and 5'-GGGTCGGAAGAC CTTAGTCC-3'; CDK2, 5'-GCCATTCTCATCGGGTCCTC
-3' and 5'-ATTTGCAGCCCAGGAGGATT-3'; cyclin D1, 5'CCGAGGAGCTGCTGCAAATGGAGCT- ${ }^{\prime}$ and $5^{\prime}$-TGAA ATCGTGCGGGGTCATTGCGGC-3'; caspase-9, 5'-GGTGA CCCCAGAATTGACCC-3' and 5'-TCGACAACTTTGCTG CTTGC-3'; Bcl-2, 5'-GGTGAACTGGGGGAGGATTG-3' and 5'-GGCAGGCATGTTGACTTCAC-3'; Bax, 5'-AGCTG AGCGAGTGTCTCAAG-3' and 5'-GTCCAATGTCCAGCC CATGA-3'; MMP9, 5'-CGCATCTGGGGCTTTAAACAT-3' and $5^{\prime}$-TCAGCACAAACAGGTTGCAG-3'; $\beta$-actin, $5^{\prime}$-TCG TGCGTGACATTAAGGAG-3' and 5'ATGCCAGGGTACATGGTGGT-3'.

\section{Statistical Analyses}

Data are the mean \pm standard deviation. Differences were evaluated by one-way analysis of variance (ANOVA) with least-square difference test. $\mathrm{P}<0.05$ was considered significant. Statistical analyses were conducted using SPSS v16.0 (IBM, Armonk, NY, USA).

\section{Results \\ Oxymatrine Repressed the Viability of BC Cells}

The BC lines MCF-7 and MDA-MB-231 and HEK-293 cells (control group) were treated by the indicated concentrations of oxymatrine. At an established time, point, oxymatrine reduced the viabilities of MCF-7 and MDA-MB-231 cells significantly in a dose-dependent manner; stronger effects were observed in MCF-7 cells, but weaker effects were seen in the control group (Figure 1B). To explore the differences between effects on these cells, we measured PI3K expression at RNA and protein levels: higher PI3K expression was noted in MCF-7 cells than in MDA-MB-231 cells, with lowest expression observed in the control group (Figure 1D and E). Oxymatrine inhibited PI3K expression in MCF-7 cells and MDA-MB-231 cells in a dose-dependent manner (Figure 1E, F, H and I).

\section{Oxymatrine Expedited the Apoptosis of BC Cells}

Hoechst 33258 staining showed that oxymatrine accelerated chromatin condensation in MCF-7 cells to induce nuclear degradation (Figure 2A). Data from flow cytometry also demonstrated that oxymatrine treatment generated more apoptotic cells $(7.63 \%$ and $1.36 \%$ at $30 \mu \mathrm{M}$ and $10 \mu \mathrm{M}$ of oxymatrine, respectively) than that in the control group (Figure 2B). Oxymatrine treatment decreased the level of Aktp-Thr308 protein, with little change in the total Akt level (Figure 2C and D). Also, oxymatrine treatment increased the expression 
A

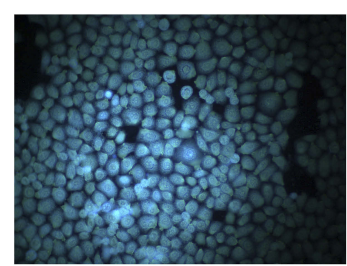

B

DMSO

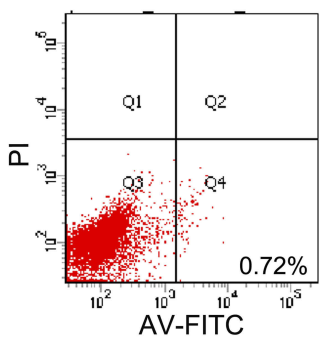

DMSO

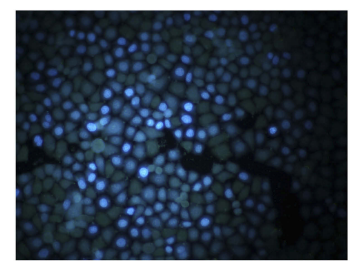

$10 \mu \mathrm{m} / \mathrm{l}$

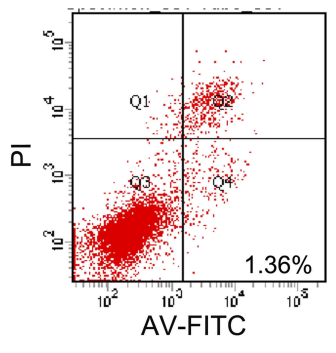

$10 \mu \mathrm{m} / \mathrm{l}$

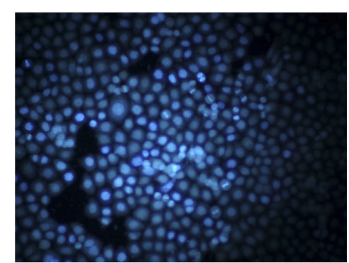

$30 \mu \mathrm{m} / \mathrm{l}$

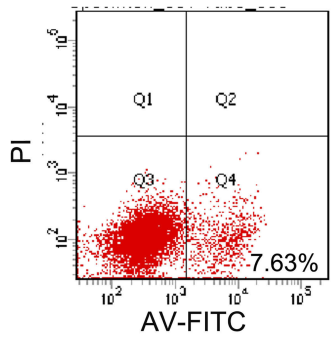

$30 \mu \mathrm{m} / \mathrm{l}$
DMSO $10 \mu \mathrm{m} / \mathrm{l} 20 \mu \mathrm{m} / \mathrm{l} 30 \mu \mathrm{m} / \mathrm{l}$

PI3K

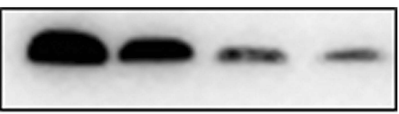

Akt

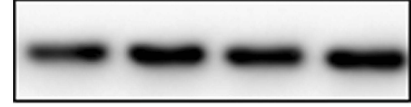

$A \mathrm{At}^{\mathrm{p}-\mathrm{Th} 308}$
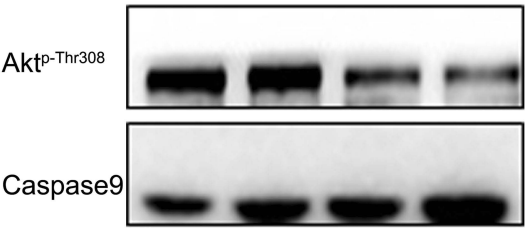

Bax

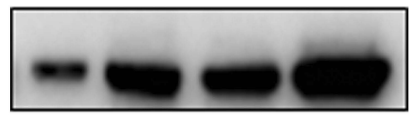

Bcl-2

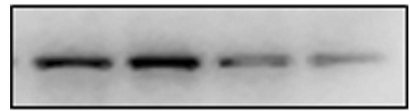

GAPDH

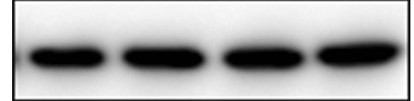

D

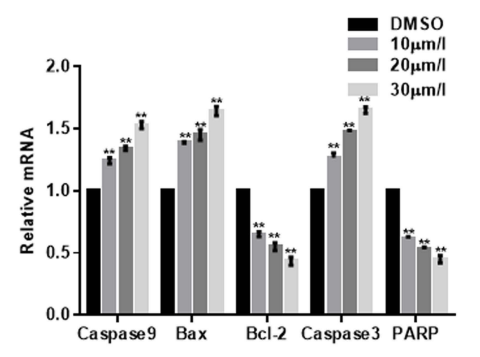

DMSO $10 \mu \mathrm{m} / \mathrm{l} 20 \mu \mathrm{m} / \mathrm{l} 30 \mu \mathrm{m} / \mathrm{l}$

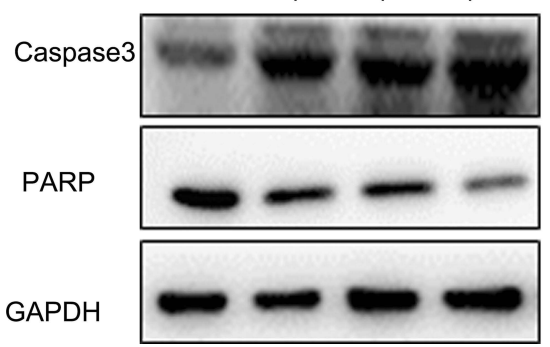

Figure 2 Oxymatrine induces the apoptosis of MCF-7 cells. (A) MCF-7 cells were pre-incubated with the indicated concentrations of oxymatrine for 24 hrs, and then cells were stained with Hoechst 33258 and observed with a fluorescence microscope. (B) MCF-7 cells were pre-incubated with the indicated concentrations of oxymatrine for $24 \mathrm{hrs}$, and cells were treated with ANNEXIN-V-FITC and analyzed by FACS analysis. The experiment was repeated thrice. (C) MCF-7 cells were treated with DMSO or the indicated concentrations of oxymatrine for 24 hrs, and expression of PI3K, AKT, Akt ${ }^{\text {-Thr308 }}$ caspase-3, Bax and Bcl-2 was measured by Western blotting. (D) MCF-7 cells were treated with DMSO or the indicated concentrations of oxymatrine for 24 hrs, and mRNA expression of caspase-3, Bax and Bcl-2 was measured by real-time RT-PCR. Results are the mean $\pm \mathrm{SD}$ of three experiments done in triplicate. ${ }^{* * P}<0.01$, compared with the control group. 
of caspase- 3 and Bax significantly at protein and RNA levels, whereas Bcl-2 expression was downregulated.

\section{Oxymatrine Suppressed Proliferation of BC Cells}

We examined the effects of oxymatrine on the cycle of MCF-7 cells by flow cytometry. Oxymatrine arrested a greater proportion of cells in the G1 phase $(67.25 \%$ and $63.24 \%$ at $30 \mu \mathrm{M}$ and $10 \mu \mathrm{M}$ of oxymatrine, respectively), and a smaller proportion in the $\mathrm{S}$ phase $(17.69 \%$ and $20.11 \%$ at $30 \mu \mathrm{M}$ and $10 \mu \mathrm{M}$ of oxymatrine, respectively) than the control group (Figure 3A). Cyclin D1, CDK2 and Gsk3 $\beta$ have central roles in regulating the $\mathrm{G} 1$ phase of the cell cycle, so we measured their expression in cells treated at different doses of oxymatrine. Western blotting showed that exposure to the indicated concentrations of oxymatrine for $48 \mathrm{hrs}$ decreased expression of cyclin D1, CDK2 and Gsk3 $\beta$ markedly (Figure 3B). Real-time PCR showed that oxymatrine inhibited the expression of cyclin D1 in a dose-dependent manner (Figure 3C). These results suggested that oxymatrine suppressed the proliferation of $\mathrm{BC}$ cells.

\section{Oxymatrine Inhibited the Migration and Invasion of BC Cells}

Transwell assays with or without Matrigel were undertaken to test the inhibitory effect of oxymatrine on the migration

A

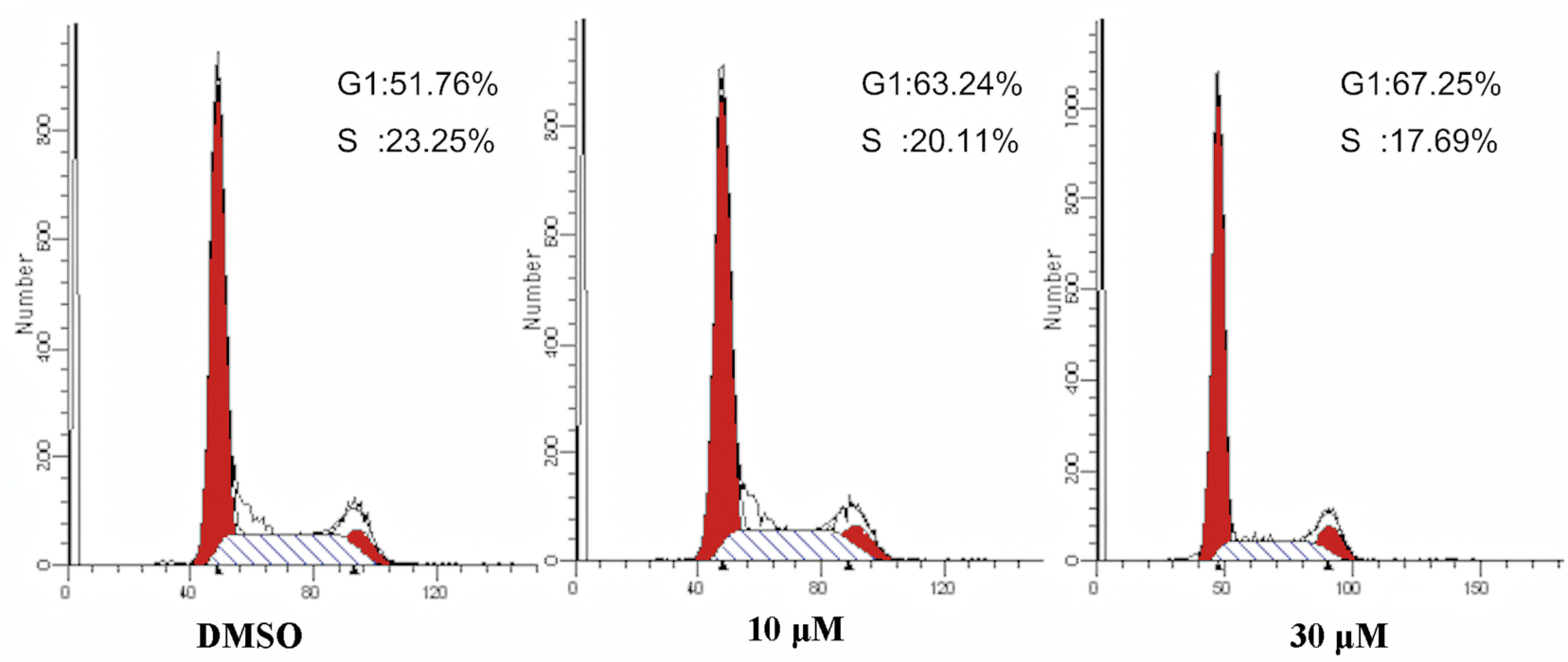

B
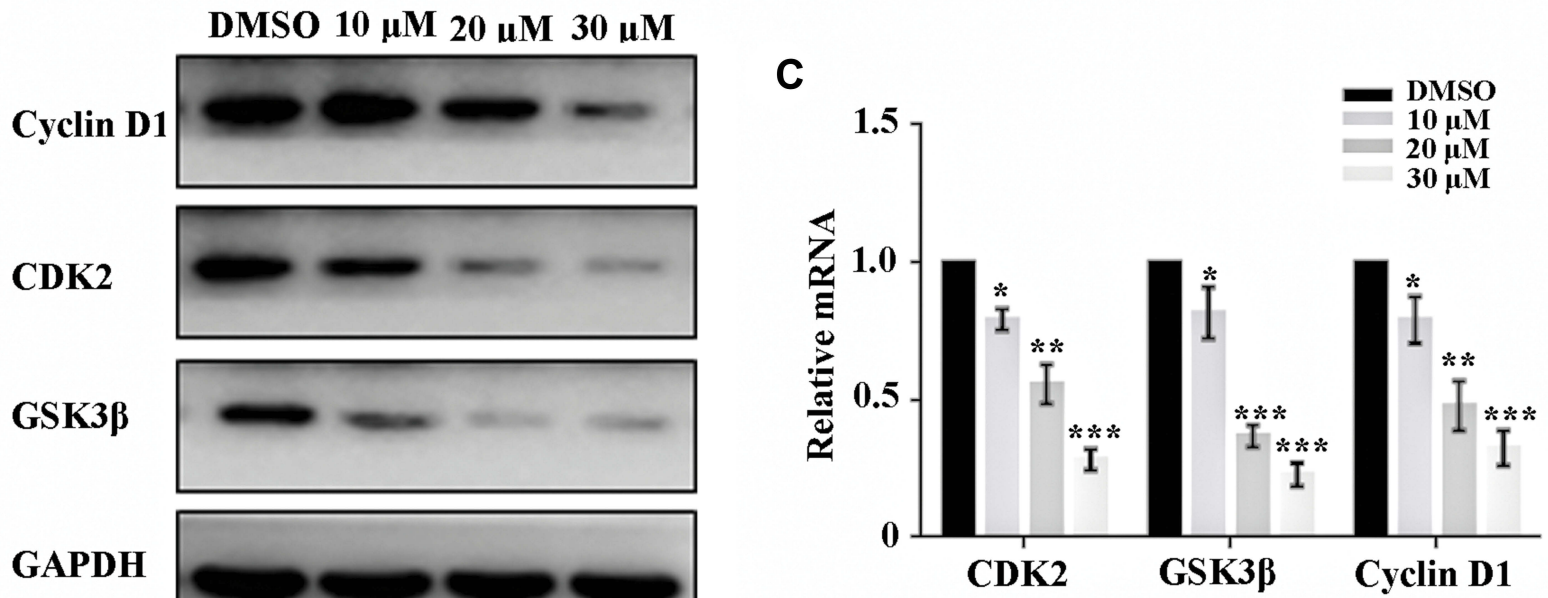

GAPDH

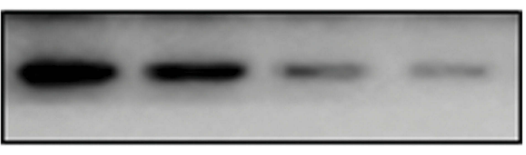

CDK2

GSK3及

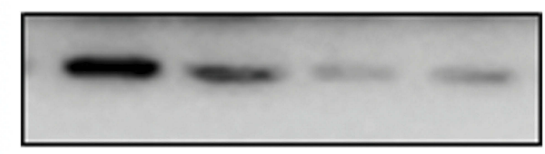

GAPDH

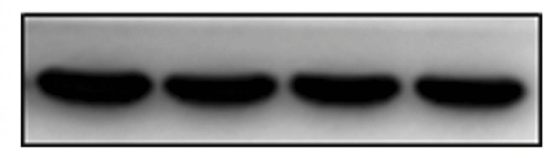

CDK2

GSK3 $\beta$

Cyclin D1

Figure 3 Oxymatrine suppressed the proliferation of breast cancer cells. (A) MCF-7 cells were pre-incubated with or without oxymatrine for 24 hrs, and then cells were analyzed using a FACS Vantage flow cytometer with CellQuest ${ }^{\mathrm{TM}}$ acquisition and analysis software. (B) MCF-7 cells were treated with DMSO or the indicated concentrations of oxymatrine for $24 \mathrm{hrs,}$ and expression of cyclin DI, CDK2 and Gsk3ß was measured by Western blotting. (C) MCF-7 cells were treated with DMSO or the indicated concentrations of oxymatrine for $24 \mathrm{hrs}$, and mRNA expression of cyclin DI, CDK2 and Gsk3ß was measured by real-time RT-PCR. Results are the mean \pm $\mathrm{SD}$. Experiments were repeated thrice. $* \mathrm{P}<0.05$, $* * \mathrm{P}<0.01$, $* * \mathrm{P}<0.001$, compared with the control group. 
A

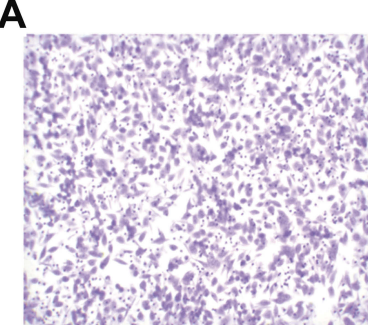

DMSO

B

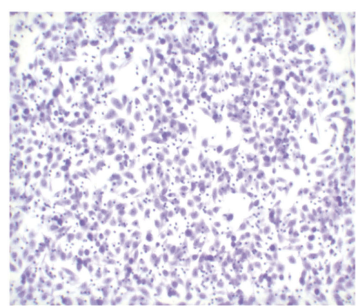

C

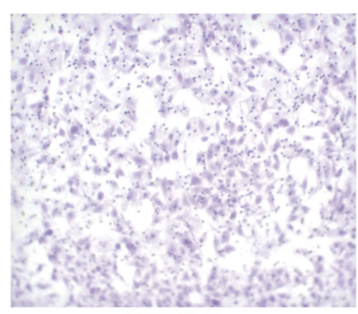

DMSO

D

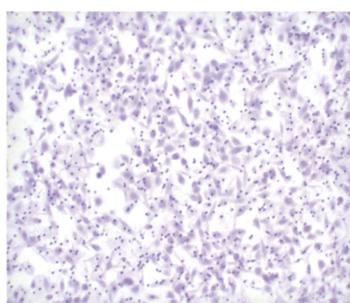

DMSO

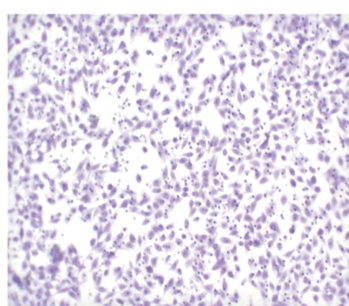

$10 \mu \mathrm{M}$

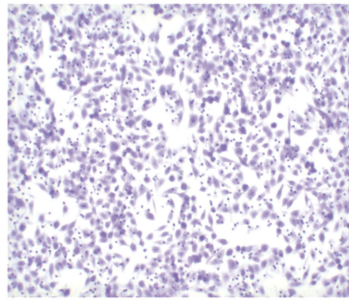

$10 \mu \mathrm{M}$

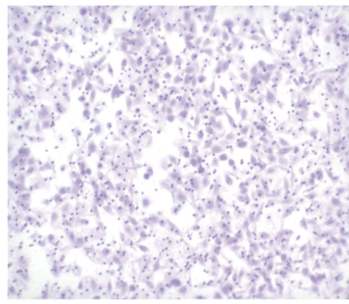

$10 \mu \mathrm{M}$

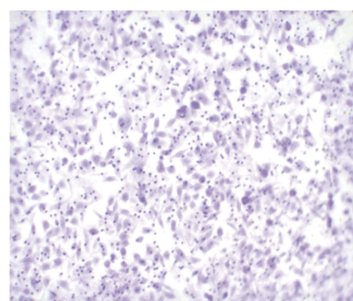

$10 \mu \mathrm{M}$

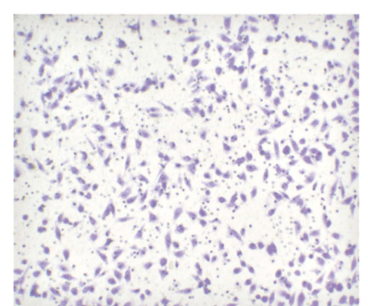

$30 \mu M$

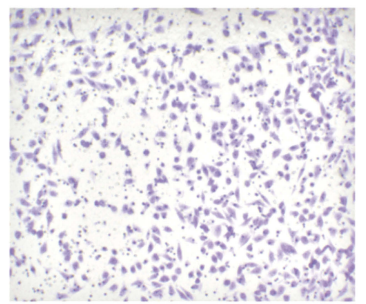

$30 \mu \mathrm{M}$

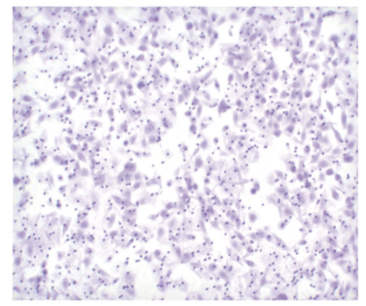

$30 \mu \mathrm{M}$

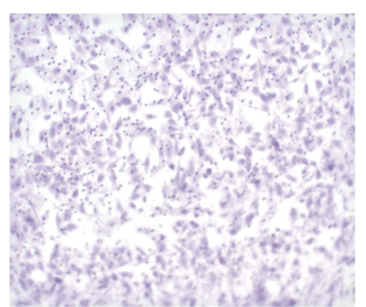

$30 \mu \mathrm{M}$

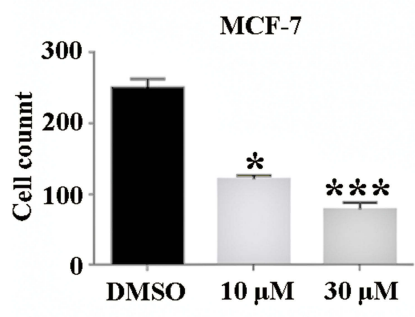

MCF-7
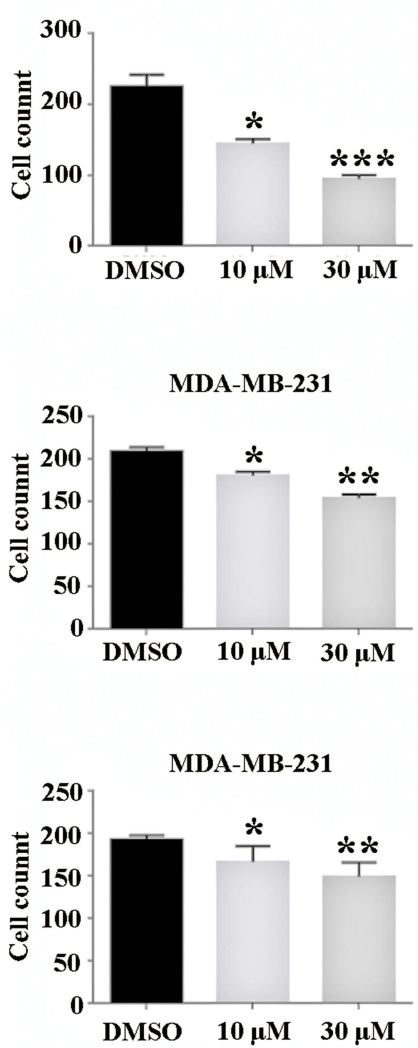

E

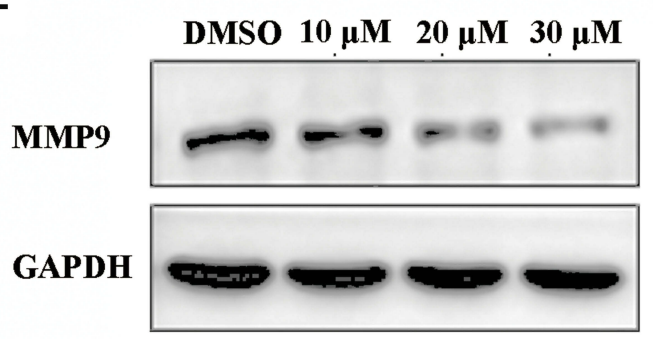

F

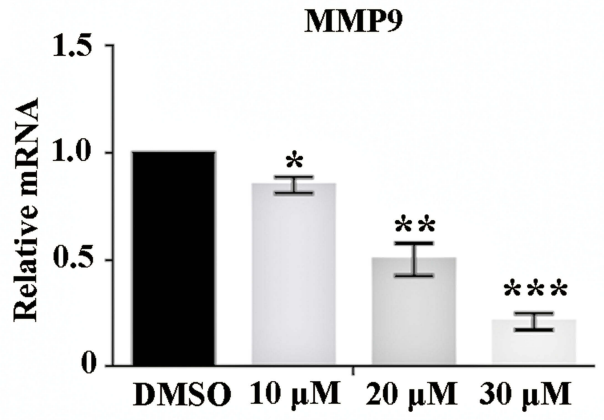

Figure 4 Oxymatrine inhibited the migration and invasion of breast cancer cells. (A and $\mathbf{B}$ ) After pre-incubation with oxymatrine, MCF-7 cells were detected by Transwell $^{\mathrm{TM}}$ without or with Matrigel ${ }^{\mathrm{TM}}$. (C and D) After pre-incubation with oxymatrine, MDA-MB-23I cells were detected by Transwell without or with Matrigel. (E) MCF-7 cells were treated with DMSO or the indicated concentrations of oxymatrine for 24 hrs, and MMP9 expression was measured by Western blotting. (F) MCF-7 cells were treated with DMSO or the indicated concentration of oxymatrine for 24 hrs, and mRNA expression of MMP9 was measured by real-time RT-PCR. Results are the mean $\pm \mathrm{SD}$. Experiments were repeated thrice. $* \mathrm{P}<0.05$, $* * \mathrm{P}<0.01$, $* * * \mathrm{P}<0.00 \mathrm{I}$, compared with the control group. 
and invasion of BC cells, respectively. Oxymatrine reduced the migratory and invasive abilities of $\mathrm{MCF}-7$ cells in a concentration-dependent manner (Figure 4A and B), whereas similar (but weaker) inhibitory effects were observed in MDA-MB-231 cells (Figure 4C and D). We demonstrated that MMP9 expression was reduced at protein and RNA levels in MCF-7 cells (Figure 4E and F).

\section{Discussion}

Oxymatrine is considered to be a novel anti-tumor agent in different types of cancer cells because it can retard proliferation and the cell cycle and induce apoptosis. However, its effects on BC cells are not known.

Here, we demonstrated that oxymatrine repressed the proliferation, migration and invasion of MCF-7 and MDAMB-231 cells efficaciously in a dose-dependent and timedependent manner. Also, we demonstrated that oxymatrine could inhibit PI3K expression.

The PI3K/Akt signaling pathway has a pivotal role in regulating the apoptosis, proliferation and motility of cells. $^{13,14}$ Also, PI3K exhibits higher expression in tumor cells than that in normal cells, suggesting that PI3K is involved in the functions of activated tumor cells, and that suppression of its expression could be an important strategy against cancer. ${ }^{15}$ Reports have shown that fangchinoline (traditional Chinese herb with anti-tumor activity) markedly inhibited proliferation of SGC7901 cells (human gastric tumor line) if high expression of PI3K occurred, but had weaker inhibitory effects on MKN45 cells if PI3K was expressed at a low level. ${ }^{16}$ In our study, higher expression of PI3K was observed in MCF-7 and MDA-MB-231 cells than that in HEK-293 cells, data that are consistent with the results showing oxymatrine to have more obvious inhibitory effects on MCF-7 and MDA-MB-231 cells than in normal cells. Furthermore, Western blotting showed that oxymatrine significantly decreased PI3K expression in a doseindependent manner. Taken together, oxymatrine exerted its anti-tumor ability via inhibition of PI3K expression.

The proliferation and division of cells are regulated in the cell cycle by complex machinery comprising cyclins and cyclin-dependent protein kinases (CDKs). ${ }^{17}$ Regulation of the G1 phase of the cell cycle has attracted attention as a target for the study and therapy of $\mathrm{BC}{ }^{18}$ Cyclin D is a downstream locus of the PI3K/Akt signaling pathway. Cyclin D forms a complex with CDK2 (or other types of $\mathrm{CDKs}$ ) to promote G1-phase progression towards the S phase. ${ }^{19}$ Consistent with those elaborations, fluorescenceactivated cell sorting (FACS) analysis in our study demonstrated that oxymatrine arrested the cycle of MCF-7 cells at the G1 phase, along with the reduced expression of cyclin D1, CDK2 and GSK3 $\beta$ in the oxymatrine-treated group, suggesting that oxymatrine suppressed proliferation of $\mathrm{BC}$ cells dramatically.

Frequently, the growth of tumor cells is due to an imbalance between cell proliferation and apoptosis. Caspase- 3 is a crucial regulator involved in apoptosis because it enhances chromatin condensation and nuclear decomposition. ${ }^{20}$ Moreover, that balance between Bax expression and Bcl-2 expression is a major factor determining the apoptotic fate of cells, ${ }^{21,22}$ and it has been an efficacious therapeutic target for $\mathrm{BC} .^{23,24}$ Thus, we measured the expression of these regulators by Western blotting: expression of caspase- 3 and Bax was downregulated after oxymatrine treatment, whereas Bcl-2 expression was upregulated in the oxymatrine-treated group.

In addition to rapid proliferation, tumor cells are characterized by metastasis by the degradation of the extracellular matrix (ECM). ${ }^{25} \mathrm{MMP}-2$ can degrade the basement membrane of the ECM to allow tumor cells to migrate out and accelerate malignant progression. ${ }^{26}$ Studies have shown that oxymatrine can reduce MMP9 expression in gastric cancer cells. ${ }^{27}$ Similarly, we showed that oxymatrine restrained the migration and invasion of MCF-7 cells significantly along with the downregulation of MMP9 expression. MMP9 is also downstream of $\mathrm{PI} 3 \mathrm{~K} / \mathrm{Akt}$, so we conjectured that oxymatrine repressed the metastasis of $\mathrm{BC}$ cells via the PI3K/Akt/MMP9 signaling pathway. Xie $\mathrm{W}$ and colleagues reported that oxymatrine can enhance the anti-tumor effects of bevacizumab against triplenegative $\mathrm{BC}$ by abating the $\mathrm{Wnt} / \beta$-catenin signaling pathway. ${ }^{28}$ Our study demonstrated that oxymatrine can also inhibit the growth of BC cells by regulating PI3K expression.

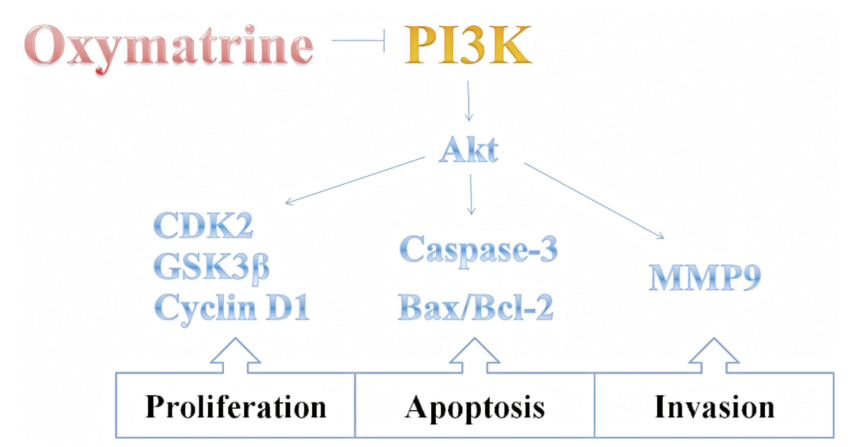

Figure 5 Suppression of proliferation and invasion and enhanced apoptosis of breast cancer cells by inhibition of expression of PI3K and its downstream signaling pathway by oxymatrine. 


\section{Conclusion}

Oxymatrine can limit the proliferation, apoptosis, migration and invasion of $\mathrm{BC}$ cells by inhibiting expression of PI3K and its downstream signaling pathways (Figure 5). However, animal models of $\mathrm{BC}$ are needed to certify the validity and safety of oxymatrine, and pharmacokinetic investigations are needed to establish its optimal dose before clinical use can be contemplated. We believe that oxymatrine could serve as a potential therapeutic agent and deserves further studies regarding anti-tumor treatment.

\section{Author Contributions}

All authors contributed to data analysis, drafting or revising the article, gave final approval of the version to be published, and agree to be accountable for all aspects of the work.

\section{Disclosure}

The authors report no conflicts of interest in this work.

\section{References}

1. Leone BA, Vallejo CT, Romero AO, et al. Prognostic impact of metastatic pattern in stage IV breast cancer at initial diagnosis. Breast Cancer Res Treat. 2017;161(3):537-548. doi:10.1007/s10549-016-4066-7

2. IARC, WHO. Global cancer observatory; 2018. Available from: http://gco.iarc.fr/today/home. Accessed November 09, 2019.

3. Yoo TG, Cranshaw I, Broom R, et al. Systematic review of early and long-term outcome of liver resection for metastatic breast cancer: is there a survival benefit? Breast. 2017;32:162-172. doi:10.1016/j. breast.2017.02.003

4. Muhammad N, Steele R, Isbell TS, et al. Bitter melon extract inhibits breast cancer growth in preclinical model by inducing autophagic cell death. Oncotarget. 2017;8:66226-66236. doi:10.18632/oncotarget.19887

5. Bhattacharya S, Muhammad N, Steele R, et al. Bitter melon enhances natural killer-mediated toxicity against head and neck cancer cells. Cancer Prev Res (Phila). 2017;10(6):337-344. doi:10.1158/19406207.CAPR-17-0046

6. Bhattacharya S, Muhammad N, Steele R, et al. Immunomodulatory role of bitter melon extract in inhibition of head and neck squamous cell carcinoma growth. Oncotarget. 2017;7(22):33202-33209. doi:10.18632/oncotarget. 8898

7. Wang X, Liu C, Wang J, et al. Oxymatrine inhibits the migration of human colorectal carcinoma RKO cells via inhibition of PAI-1 and the TGF- $\beta 1 /$ Smad signaling pathway. Oncol Rep. 2017;37 (2):747-753. doi:10.3892/or.2016.5292

8. Chen H, Zhang J, Luo J, et al. Antiangiogenic effects of oxymatrine on pancreatic cancer by inhibition of the NF- $\mathrm{kB}$-mediated VEGF signaling pathway. Oncol Rep. 2013;30(2):589-595. doi:10.3892/ or.2013.2529

9. Zou J, Ran ZH, Xu Q, et al. Experimental study of the killing effects of oxymatrine on human colon cancer cell line SW1116. Chin J Dig Dis. 2005;6(1):15-20. doi:10.1111/j.1443-9573.2005.00181.x

10. Liu Y, Bi T, Dai W, et al. RETRACTED: effects of oxymatrine on the proliferation and apoptosis of human hepatoma carcinoma cells. Technol Cancer Res Treat. 2016;15(3):487-489. doi:10.1177/1533034615587616
11. Guo B, Zhang T, Su J, et al. Oxymatrine targets EGFR(p-Tyr845) and inhibits EGFR-related signaling pathways to suppress the proliferation and invasion of gastric cancer cells. Cancer Chemother Pharmacol. 2015;75(2):353-363. doi:10.1007/s00280-014-2651-1

12. Zhang Y, Sun S, Chen J, et al. Oxymatrine induces mitochondria dependent apoptosis in human osteosarcoma MNNG/HOS cells through inhibition of PI3K/Akt pathway. Tumour Biol. 2014;35 (2):1619-1625. doi:10.1007/s13277-013-1223-z

13. Safdari Y, Khalili M, Ebrahimzadeh MA, et al. Natural inhibitors of $\mathrm{PI} 3 \mathrm{~K} / \mathrm{AKT}$ signaling in breast cancer: emphasis on newly-discovered molecular mechanisms of action. Pharmacol Res. 2015;93:1-10. doi:10.1016/j.phrs.2014.12.004

14. Chia S, Gandhi S, Joy AA, et al. Novel agents and associated toxicities of inhibitors of the pi3k/Akt/mTOR pathway for the treatment of breast cancer. Curr Oncol. 2015;22(1):33-48. doi:10.3747/co.22.2393

15. Mayer IA, Arteaga CL. The PI3K/AKT pathway as a target for cancer treatment. Annu Rev Med. 2016;67:11-28. doi:10.1146/ annurev-med-062913-051343

16. Tian F, Ding D, Li D. Fangchinoline targets PI3K and suppresses PI3K/AKT signaling pathway in SGC7901 cells. Int $J$ Oncol. 2015;46(6):2355-2363. doi:10.3892/ijo.2015.2959

17. Casimiro MC, Velasco-Velázquez $\mathrm{M}$, Aguirre-Alvarado $\mathrm{C}$, et al. Overview of cyclins D1 function in cancer and the CDK inhibitor landscape: past and present. Expert Opin Investig Drugs. 2014;23 (3):295-304. doi:10.1517/13543784.2014.867017

18. Xing Z, Zhang Y, Zhang X, et al. Fangchinoline induces G1 arrest in breast cancer cells through cell-cycle regulation. Phytother Res. 2013;27(12):1790-1794. doi:10.1002/ptr.4936

19. Takahashi-Yanaga F, Sasaguri T. GSK-3 $\beta$ regulates cyclin D1 expression: a new target for chemotherapy. Cell Signal. 2008;20(4):581-589. doi:10.1016/j.cellsig.2007.10.018

20. Shalini S, Dorstyn L, Dawar S, et al. Old, new and emerging functions of caspases. Cell Death Differ. 2015;22(4):526-539. doi:10.1038/ cdd.2014.216

21. Zeng C, Ke Z, Song Y, et al. Annexin A3 is associated with a poor prognosis in breast cancer and participates in the modulation of apoptosis in vitro by affecting the Bcl-2/Bax balance. Exp Mol Pathol. 2013;95(1):23-31. doi:10.1016/j.yexmp.2013.04.002

22. Sharifi S, Barar J, Hejazi MS, et al. Doxorubicin changes Bax/Bcl-xL ratio, caspase-8 and 9 in breast cancer cells. Adv Pharm Bull. 2015;5 (3):351-359. doi:10.15171/apb.2015.049

23. Vafaiyan Z, Gharaei R, Asadi J. The correlation between telomerase activity and Bax/Bcl-2 ratio in valproic acid-treated MCF-7 breast cancer cell line. Iran J Basic Med Sci. 2015;18(7):700-704.

24. Kordezangeneh M, Irani S, Mirfakhraie R, et al. Regulation of BAX/ BCL2 gene expression in breast cancer cells by docetaxel-loaded human serum albumin nanoparticles. Medical Oncology. 2015;32 (7). doi:10.1007/s12032-015-0652-5

25. Das S, Banerji A, Frei E, et al. Rapid expression and activation of MMP-2 and MMP-9 upon exposure of human breast cancer cells (MCF-7) to fibronectin in serum free medium. Life Sci. 2008;82(9-10):467-476. doi:10.1016/j.1fs.2007.12.013

26. Nilsson UW, Garvin S, Dabrosin C. MMP-2 and MMP-9 activity is regulated by estradiol and tamoxifen in cultured human breast cancer cells. Breast Cancer Res Treat. 2007;102(3):253-261. doi:10.1007/ s10549-006-9335-4

27. Guo B, Zhang T, Su J, et al. Oxymatrine targets EGFRp-Tyr845 and inhibits EGFR-related signaling pathways to suppress the proliferation and invasion of gastric cancer cells. Cancer Chemother Pharmacol. 2017;75(2):353-363. doi:10.1007/s00280-014-2651-1

28. Xie W, Zhang Y, Zhang S, et al. Oxymatrine enhanced anti-tumor effects of Bevacizumab against triple-negative breast cancer via abating Wnt/ $\beta$ Catenin signaling pathway. Am J Cancer Res. 2019;9(8):1796-1814. 


\section{Publish your work in this journal}

Cancer Management and Research is an international, peer-reviewed open access journal focusing on cancer research and the optimal use of preventative and integrated treatment interventions to achieve improved outcomes, enhanced survival and quality of life for the cancer patient.
The manuscript management system is completely online and includes a very quick and fair peer-review system, which is all easy to use. Visit http://www.dovepress.com/testimonials.php to read real quotes from published authors.

Submit your manuscript here: https://www.dovepress.com/cancer-management-and-research-journal 